\title{
Complete Disappearance of Numerous Hepatic and Adrenal Metastases after One Dose of Pembrolizumab in a Patient with Metastatic Malignant Melanoma
}

\author{
Muhammad Zubair Afzal ${ }^{1, *}$, Keisuke Shirai ${ }^{2}$ \\ ${ }^{1}$ Hospital Medicine, Dartmouth-Hitchcock Medical Center, One Medical Center Dr. Lebanon, NH, 03756 \\ ${ }^{2}$ Hematology-Oncology, Norris Cotton Cancer Center, One Medical Center Dr. Lebanon, NH, 03756 \\ *Corresponding author: Muhammad.afzal@hitchcock.org
}

Received January 02, 2019; Revised February 09, 2019; Accepted February 10, 2019

\begin{abstract}
Although pembrolizumab is the mainstay treatment in metastatic malignant melanoma and have shown a durable response. Heterogeneous or no response has been observed in $30-40 \%$ of the cases of metastatic malignant melanoma. Metastatic lung lesions are sensitive to checkpoint inhibitor therapy whereas liver and adrenal metastases show a variable response. We are reporting a case of malignant melanoma with numerous liver and adrenal metastases showing a significant and durable response to just a single dose of pembrolizumab.
\end{abstract}

Keywords: metastatic malignant melanoma, pembrolizumab, adrenal metastasis, hepatic metastasis

Cite This Article: Muhammad Zubair Afzal, and Keisuke Shirai, "Complete Disappearance of Numerous Hepatic and Adrenal Metastases after One Dose of Pembrolizumab in a Patient with Metastatic Malignant Melanoma." American Journal of Medical Case Reports, vol. 7, no. 2 (2019): 22-23. doi: 10.12691/ajmcr-7-2-2.

\section{Letter}

Respected Editor,

Pembrolizumab is one of the immune checkpoint inhibitors (ICI) and is a monoclonal antibody against programmed cell death protein -1 (PD-1). It was approved by FDA in 2014 based on the results of phase $1 \mathrm{~b}$ KEYNOTE -001 clinical trial [1]. Although, rate of fatal toxicities by ICI is $0.3-1.3 \%$ of the treated patients [2], currently, ICI especially the pembrolizumab is the mainstay treatment of stage IIIC or IV metastatic malignant melanoma due to considerably improved overall survival (OS) and disease related outcomes. Although, pembrolizumab have shown durable response in malignant melanoma, studies have showen hetrogeneous response or no response at all in about $30 \%-40 \%$ of the cases $[3,4]$. A higher response to pembrolizumab have been shown for metastatic lesions to the lungs, whereas a variable response have been shown in case of liver and adrenal metastases in malignant melanoma [5,6,7]. Here we are reporting a complete dissappearance of numerous hepatic and adrenal metastases after a single dose of pembrolizumab in malignant melanoma patient.

This is an 87 year old man with a history of marginal zone non-hodgkin lympoma (NHL) of the thyroid, prostate cancer (status-post prostatectomy 17 years ago), remote history of cutaneous melanoma on his back and face. Ten years later, the patient presented with vague abdominal pain. CT scan of the abdomen revealed multiple liver lesions. MRI of the abdomen suggested a metastatic disease. Given history of NHL, and elevated $\mathrm{LDH}$, initially it was thought to be the recurrence of his lymphoma but the liver biopsy was consistent with metastatic melanoma (BRAF/c-kit/NRAS mutation negative, MLH1 mutation positive, TP53 mutation positive). MRI of the brain was negative for intracranial metastasis. A PET/CT scan showed disffuse liver and adrenal metastases [Figure 1A]. Patient was stated on pembrolizumab. The patient developed thrombocytopenia $\left(60 \times 10^{3}\right)$ and loss of appetitie after first cycle of pembrolizumab. As a result, cycle 2 was held. Patient continued to have thrombocytopenia (albiet improvement in overall platelet count upto $90 \times 10^{3}$ ), and we continued to hold pembrolizumab therapy, as a CT scan 6 weeks after first dose of pembrolizumab showed partial response in liver and adrenal lesions. Lactate dehydrogenase (LDH) decreased from 1293 unit/L to 262 unit/L as well. Given partial response (PR) on CT scan, improving LDH, liver function tests (LFT's) and persistent but stable thrombocytopenia, while patient was doing clinically better, we opted for survillance. A survillance PET/CT scan done 3 months after the only cycle of pembrolizumab, showed no obvious disease activity in adrenal gland and liver [Figure 1B]. At the time of this report, the patient is one year since the only cycle of pembrolizumab and continued to have stable disease after initial significant response. 


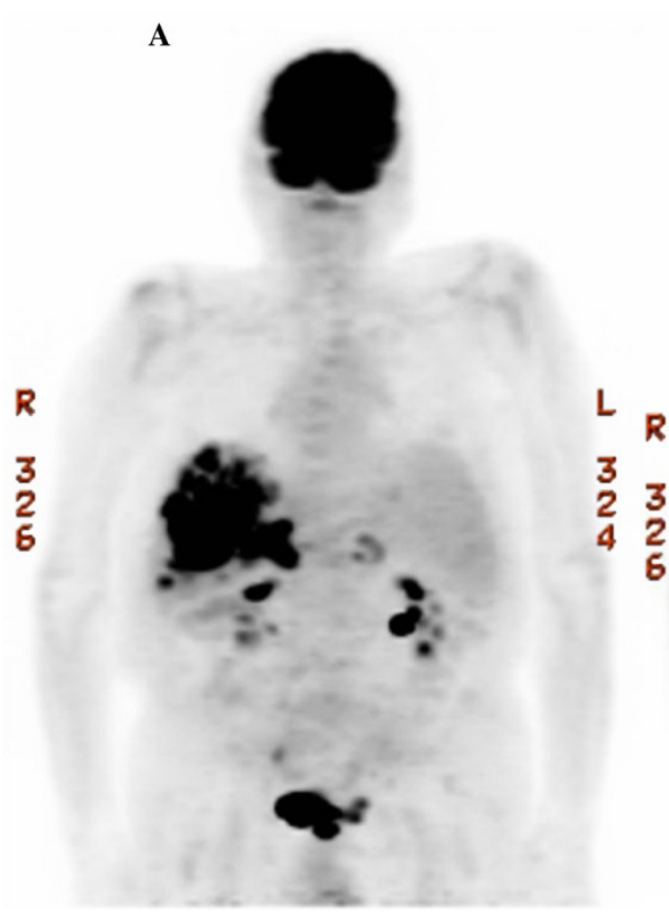

B

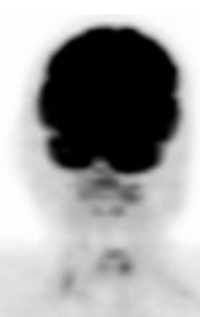

$\begin{array}{ll}L & R \\ 3 & \\ 2 & 3 \\ 4 & 2 \\ & 6\end{array}$ $\mathbf{L}$

Figure 1. $\mathrm{A}=$ Pre-treatment PET/CT showing metastatic lesions in liver and adrenal gland, $\mathrm{B}=$ Disappearance of metastatic lesions after one cycle of pembrolizumab

\section{Funding Source}

None.

\section{Acknowledgements}

Not Available.

\section{Conflict of Interest}

None declared by both authors

\section{Source of Funding}

Not Available

\section{Declaration of Patient Consent}

We have obtained consent from the patient. The patients understand that their names and initials will not be published, and due efforts will be made to conceal their identity.

\section{References}

[1] Garon EB, Rizvi NA, Hui R, et al. Pembrolizumab for the Treatment of Non-Small-Cell Lung Cancer. New England Journal of Medicine, 2015; 372(21), 2018-2028.

[2] Wang DY, Salem J, Cohen JV. Fatal Toxic Effects Associated With Immune Checkpoint Inhibitors. JAMA Oncology. 2018.

[3] Ribas A, Hamid O, Daud A, et al. Association of pembrolizumab with tumor response and survival among patients with advanced melanoma. JAMA 2016; 315: 1600-1609.

[4] Robert C, Schachter J, Long GV, et al. Pembrolizumab versus ipilimumab in advanced melanoma. N Engl J Med 2015; 372: 2521-2532.

[5] Khoja L, Kibrio M, Metser U, et al. Patterns of response to antiPD-1 treatment: an exploratory comparison of four radiological response criteria and associations with overall survival in metastatic melanoma patients. Br J Cancer 2016; 115: 1186-1192.

[6] Tumeh PC, Harview CL, Yearley JH, et al. PD-1 blockade induces responses by inhibiting adaptive immune resistance. Nature 2014; 515: 568-571

[7] Nguyen MC, Shah MH, Liebner DA, et al. The Adrenal Gland as a Sanctuary Site of Metastases After Pembrolizumab Treatment: A Case Series. Journal of the National Comprehensive Cancer Network, 2018; 16(11), 1279-1283.

C The Author(s) 2019. This article is an open access article distributed under the terms and conditions of the Creative Commons Attribution (CC BY) license (http://creativecommons.org/licenses/by/4.0/). 